\title{
Exposure Time Point Reference
}

National Cancer Institute

\section{Source}

National Cancer Institute. Exposure Time Point Reference. NCI Thesaurus. Code C83291.

The point in time that acts as a fixed reference point to an exposure assessment. 